\title{
LETIZIA PELLEGRINI
}

\section{On the industrial and mechanical applications of dual variables in linear programming}

Revue française d'automatique, d'informatique et de recherche opérationnelle. Recherche opérationnelle, tome 32, nº 4 (1998), p. 425-435.

<http://www.numdam.org/item?id=RO_1998_32_4_425_0>

(C) AFCET, 1998, tous droits réservés.

L'accès aux archives de la revue « Revue française d'automatique, d'informatique et de recherche opérationnelle. Recherche opérationnelle » implique l'accord avec les conditions générales d'utilisation (http://www.numdam.org/ legal.php). Toute utilisation commerciale ou impression systématique est constitutive d'une infraction pénale. Toute copie ou impression de ce fichier doit contenir la présente mention de copyright.

\section{Numdam}

Article numérisé dans le cadre du programme

Numérisation de documents anciens mathématiques

http://www.numdam.org/ 


\title{
ON THE INDUSTRIAL AND MECHANICAL APPLICATIONS OF DUAL VARIABLES IN LINEAR PROGRAMMING (*)
}

\author{
by Letizia Pellegrini $\left({ }^{1}\right)$ \\ Communicated by Franco GiANNESSI
}

\begin{abstract}
The interpretation of a linear program in an industrial context is a well-known fact: it models a problem of maximum profit with scarce resources and the optimal solution of its dual problem is interpreted as a shadow prices vector. Nevertheless, as pointed out by Dantzig and Jackson in [3], this classic interpretation comes short when one of the resources is not completely exhausted in the optimal production process; in fact, in such case, its value is set to zero. In this paper a generalization of the improvement based on a perturbation method and proposed by Dantzig and Jackson is analysed, discussed and compared with another approach [1]; it is proved that such a perturbation can lead to a situation where dual variables do not allow any evaluation of the resources. This inadequacy is brought up every time we apply Linear Programming in order to model a real problem: in this paper this shortcoming is presented and discussed in a primal-dual pair describing a classic problem of Mechanics. (c) Elsevier, Paris
\end{abstract}

Keywords: Dual variables, shadow prices.

Résumé. - L'interprétation d'un problème de programmation linéaire dans un contexte industriel est bien connue : il représente un problème de profit maximal avec de maigres ressources et la solution optimale de son problème dual est interprétée comme un vecteur de prix ombre. Néanmoins, comme Dantzig et Jackson l'ont remarqué dans [3], cette interprétation classique montre un défaut quand une des ressources n'est pas complètement employée dans le procédé optimal de production; en effet, dans ce cas sa valeur est imposée égale à zéro. Dans cet article on analyse et on discute une généralisation de la solution basée sur une méthode de perturbation et proposée par Dantzig et Jackson tout en la comparant avec une approche différente [1]. On démontre que dans certains cas cette perturbation peut conduire à une situation dans laquelle les variables duales ne permettent pas d'évaluer les ressources. Cette inadéquation se présente chaque fois qu'on utilise la Programmation Linéaire pour modéliser un problème réel : dans cet article cette inadéquation est présentée et discutée en utilisant une paire primale-duale qui décrit un problème classique de Mécanique. (c) Elsevier, Paris

Mots clés : Variables duales, prix ombre.

\section{INTRODUCTION}

Let us consider a linear programming problem in the following format:

$$
\max c^{T} x, \quad \text { s.t. } A x \leq b, \quad x \geq 0,
$$

(*) Manuscript received July 1996 .

( $\left.{ }^{1}\right)$ Associate Professor, Institute of Mathematics, Faculty of Economics, University of Verona, Via dell'Artigliere 19, 37129 Verona, Italy.

Recherche opérationnelle/Operations Research, 0399-0559/98/04/

(C) Elsevier, Paris 
where $A=\left(a_{i j}\right), i=1, \ldots, m ; j=1, \ldots, n$ is a matrix of order $m \times n$, $c^{T}=\left(c_{1}, \ldots, c_{n}\right)$ a row $n$-vector, $b$ a column $m$-vector, all with real entries, and $T$ denotes transposition. In the sequel, $A_{r}$ will denote the $r$-th row of $A$ and $b_{r}$ the $r$-th element of $b$; "s.t." means "subject to".

Problem (1.1) is called primal problem, when it is associated with the dual problem, which is another linear program in the following format:

$$
\min \lambda^{T} b, \quad \text { s.t. } \quad \lambda^{T} A \geq c^{T}, \quad \lambda \geq 0,
$$

where $\lambda^{T}=\left(\lambda_{l}, \ldots, \lambda_{m}\right)$ is a row $m$-vector with real entries.

In an industrial context, an interpretation of (1.1) is a well-known fact: it arises when $A$ is the so-called technological matrix, $c$ the vector of incomes and $b$ the vector of available factors of production (i.e., the resources); $x$ is the unknown vector of outputs. Consequently, even the dual of (1.1) has a classic interpretation as shown below.

The industry, instead of transforming the resources $b$ into the outputs $x$, can, hypothetically, consider the conditions under which it should be more convenient to sell the resources directly (instead of transforming them into goods); this leads to problem (1.2): any existing feasible solution would make the direct selling of resources more convenient or at least not worse than their transformation in final consumption goods. The minimum point $\lambda^{0}$ of (1.2) is the vector of so-called shadow prices of the $m$ resources; it represents the worst situation of this second model and, as implied by the Duality Theorem for Linear Programming [2], leads in (1.2) to the same optimal value of (1.1), i.e. to economic equilibrium.

In 1978 Dantzig and Jackson [3] made the following important remark. If a maximum point of (1.1), say $x^{0}$, is such that:

$$
A_{r} x^{0}<b_{r},
$$

then, because of the well-known Complementarity Slackness Theorem [2], it results that $\lambda_{r}^{0}=0$; in other words, if a resource is not completely expended in the production process, its value is set to zero. This is obviously in contrast with the real situation, where a limited resource, if employed in a positive quantity, receives a positive quantification, even in the case of a surplus in the production process. Hence the classic model fails.

This shortcoming will be analysed in this paper by exploiting the properties of primal and dual problems. Moreover, the rectification proposed in [3] and 
based on a particular perturbation technique will be examined, discussed and compared together with another approach [1], by proving that a generalization of the perturbation technique can lead to a situation where dual variables do not allow any evaluation of the resources. The inadequacy of the classic model (and consequently the failure of the perturbation technique) is not a peculiarity of the industrial problem, but is present every time we apply the Linear Programming in order to model a real problem: for example, it will be shown that a similar "pathology" is present in a primal-dual pair describing a classic and elementary problem of Mechanics.

\section{RELATIONSHIPS BETWEEN PRIMAL AND DUAL PROBLEMS}

The Duality Theory [2] establishes important relationships between a primal problem and its dual.

Let us consider problem (1.1) with the addition, in $A x \leq b$, of slack non-negative variables, whose row vector is $y^{T}=\left(y_{1}, \ldots, y_{m}\right)$. If the $(m+n)$-vector $\left(x^{0^{T}}, y^{0^{T}}\right)=\left(x_{B}^{0^{T}}, x_{N}^{0^{T}}\right)$ is an optimal non-degenerate basic solution of (1.1) and $(A, I)=(B, N)$ is the corresponding (unique) decomposition into basic and non-basic submatrices, then $\left(\lambda^{0}\right)^{T}=c_{B}^{T} B^{-1}$ is a minimum point of (1.2); this allows to express the maximum of (1.1) in terms of $b$, i.e.

$$
G(b):=\max _{\substack{A x+y=b \\(x, y) \geq 0}} c^{T} x=c_{B}^{T} B^{-1} b,
$$

where $\left(c_{B}^{T}, c_{N}^{T}\right)$ is the partitioning of $\left(c^{T}, 0\right)$ in a accordance with that of $(A, I)=(B, N)$. It follows that the minimum point of (1.2) is the gradient of $G(b)$ :

$$
\frac{\partial G(b)}{\partial b_{i}}=\left(c_{B}^{T} B^{-1}\right)_{i}=\lambda_{i}^{0}, \quad i=1, \ldots m .
$$

Hence $\lambda^{0}$ may be interpreted as the "instantaneous velocity" of the maximum function $G(b)$ with respect to a variation of $b$. In the industrial context these velocities, being the rates of change of the maximum function with respect to the vector $b$ of available factors of production, are assumed to be the shadow prices of the $m$ resources.

Unfortunately, this assumption is not correct when (1.3) occurs, i.e., when the slack variable $y_{r}$ is positive, so that the corresponding value of $\lambda_{r}^{0}$ collapses to zero. In this case, in [1] a proposal has been made to obtain 
a non-zero shadow price associated to the $r$-th resource. Such a proposal consists in replacing in (1.1) the $r$-th constraint with $A_{r} x \leq \bar{b}_{r}$, where $\bar{b}_{r}:=A_{r} x^{0}$; this means that the $r$-th resource is reduced in such a way that its use in the optimal production process is exactly equal to its availability.

Consider the following problem:

$$
\max c^{T} x, \quad \text { s.t. } \quad A x+y=\bar{b}, \quad(x, y) \geq 0,
$$

where $\bar{b}^{T}=\left(b_{1}, \ldots, b_{r-1}, \bar{b}_{r}, b_{r+1}, \ldots, b_{m}\right)$; it turns out that $\left(x^{0^{T}}, y^{0^{T}}\right)$ is a degenerate basic optimal solution of (2.1). Hence, there are $h \geq 1$ optimal basic matrices $B_{i}, i=1, \ldots, h$; each of these bases characterizes a dual optimal solution, say $\lambda^{0 i}$. Since it results that $\left(\lambda^{0 i}\right)^{T}=c_{B_{i}}^{T} B_{i}^{-1}$, $i=1, \ldots, h$, the $r$-th element of every vector $\lambda^{01}, \ldots, \lambda^{0 h}$ may be assumed to be a shadow price of the $r$-th resource; in fact, since there are $h$ different ways (i.e., $h$ different bases) for identifying the production strategy $x^{0}$, then we meet not necessarily a unique evaluation of the $r$-th price. It is important to observe that in this case the function $G(b)$ is not necessarily differentiable at $\bar{b}$.

The evaluation can be even more general. Let us consider in $\mathbb{R}_{+}^{m}$ the convex hull of $\lambda^{01}, \ldots, \lambda^{0 h}$; it is a subset of a face, say $F$, of the feasible set of the dual of (2.1). Every point of this face $\left(\lambda^{01}, \ldots, \lambda^{0 h}\right.$ included) is an optimal solution of the dual of (2.1); hence it can be chosen as vector of shadow prices and, in particular, the $r$-th element of every vector of the face $F$ can be interpreted as price of the $r$-th resource.

Generally, a function $\mu: F \subseteq \mathbb{R}_{+}^{m} \rightarrow \mathbb{R}_{+}^{m}$ can be defined in such a way that $\mu_{r}(\lambda), \lambda \in F$, is the price of the $r$-th resource.

\section{A PERTURBATION METHOD}

In [3] Dantzig and Jackson made the observation that suggested the procedure explained in Section 2; moreover, they also proposed a solution based on a particular perturbation technique.

Following their suggestion, we introduce, starting from problem (2.1), a perturbed problem, called $P(\varepsilon)$ because of its dependence on a parameter $\varepsilon$. The idea consists in employing the $\varepsilon$ perturbation in such a way that the optimal solution of $P(\varepsilon)$ is non-degenerate, and subsequently studying the optimal solution of the primal problem and the limits of the corresponding dual solutions as $\varepsilon$ goes to zero, i.e. as the perturbation vanishes. 
We will show that the above perturbation method does not enlarge -with respect to the proposal made in [1]- the possibilities of evaluation of shadow prices; and even, an example will prove that, by using a general perturbation method (and not only the above special one), it is possible to obtain a situation where no evaluation is allowed.

Let us consider an optimal basic solution of (1.1), say $\left(x^{0^{T}}, y^{0^{T}}\right)$. Since $\bar{b}_{r}=A_{r} x^{0}<b_{r}$, then $\left(x^{0^{T}}, y^{0^{T}}\right)$ is an optimal basic solution of (2.1) too; however it is degenerate because $x^{0}$ satisfies at least $n+1$ constraints as equalities, while exactly $n$ of these constraints identify $x^{0}$ in a non-degenerate way.

Further, we will refer to (2.1) instead of $(1.1) ; \bar{b}$ will be replaced by $b$ and (2.1) will be called $P$.

Let us consider a perturbation of the $m$ constraints $A x \leq b$ and define the problem $P(\varepsilon)$ :

$$
\max c^{T} x, \quad \text { s.t. } \quad A x+y=b(\varepsilon), \quad(x, y) \geq 0,
$$

where $b(\varepsilon)=b-\varepsilon$ and $\varepsilon^{T}:=\left(\varepsilon_{1}, \ldots, \varepsilon_{m}\right) \geq 0$. Obviously, if $\varepsilon_{i}=0$ then the $i$-th constraint is not perturbed; hence the number of constraints effectively perturbed is $\leq m$.

Let us suppose that the maximum point $\left(x^{0^{T}}, y^{0^{T}}\right)$ of $(2.1)$ is unique and call $\left(x^{T}(\varepsilon), y^{T}(\varepsilon)\right)$ an optimal solution of problem $P(\varepsilon)$. Among all possible ways of perturbing the problem, we will consider only those for which $\left(x^{T}(\varepsilon), y^{T}(\varepsilon)\right)$ is the unique optimal solution of $P(\varepsilon)$ and is individuated by one of the $h$ bases which correspond to $\left(x^{0^{T}}, y^{0^{T}}\right)$.

Obviously, by the definition of $P(\varepsilon)$, it results that $\lim _{\varepsilon \downarrow 0} P(\varepsilon)=P$ and moreover the following proposition holds.

PRoposition 3.1: It results that $\lim _{\varepsilon \downarrow 0}\left(x^{T}(\varepsilon), y^{T}(\varepsilon)\right)=\left(x^{0^{T}}, y^{0^{T}}\right)$

Proof: For every $\varepsilon>0$ there exists a partitioning of $(A, I)$, say $\left(B_{\varepsilon}, N_{\varepsilon}\right)$, such that $\left(x^{0^{T}}, y^{0^{T}}\right)=\left(\left(B_{\varepsilon}^{-1} b\right)^{T}, 0\right)$ and such that

$$
\left(x^{T}(\varepsilon), y^{T}(\varepsilon)\right)=\left(\left(B_{\varepsilon}^{-1} b(\varepsilon)\right)^{T}, 0\right) .
$$

Since $\lim _{\varepsilon \downarrow 0} b(\varepsilon)=b$, then - whatever $B_{\varepsilon}$ may be - it follows that

$$
\begin{aligned}
\lim _{\varepsilon \downarrow 0}\left(x^{T}(\varepsilon), y^{T}(\varepsilon)\right) & =\lim _{\varepsilon \downarrow 0}\left(\left(B_{\varepsilon}^{-1} b(\varepsilon)\right)^{T}, 0\right) \\
& =\left(\left(B_{\varepsilon}^{-1} b\right)^{T}, 0\right)=\left(x^{0^{T}}, y^{0^{T}}\right) .
\end{aligned}
$$


The continuity of the minimum of $P(\varepsilon)$ follows from Proposition 3.1; in fact, it turns out that $\lim _{\varepsilon \downarrow 0} c^{T} x(\varepsilon)=c^{T} x^{0}$.

Let $P^{*}$ and $P^{*}(\varepsilon)$ be the dual problems of $P$ and $P(\varepsilon)$, respectively; obviously, it results that $\lim _{\varepsilon \downarrow 0} P^{*}(\varepsilon)=P^{*}$. Moreover, let $\lambda(\varepsilon)$ be the optimal solution of $P^{*}(\varepsilon)$ (that is unique under the above hypotheses), while - as already observed - there is no uniqueness for the optimal solution of $P^{*}$. The continuity of the optimal value also holds for the dual problem; in fact, from the Duality Theorem for Linear Programming [2], it results that:

$$
\lim _{\varepsilon \downarrow 0} \lambda^{T}(\varepsilon) b(\varepsilon)=\lim _{\varepsilon \downarrow 0} c^{T} x(\varepsilon)=c^{T} x^{0},
$$

where $c^{T} x^{0}$ is equal to the optimal value of $P^{*}$, given by all $h$ bases or equivalently by all $h$ optimal solutions of $P^{*}$. However, $\lim _{\varepsilon \downarrow 0} \lambda(\varepsilon)$ may not exist, as shown in the following example.

Example 3.1: Let us consider the problem $P$ :

$$
\max \left(3 x_{1}+x_{2}\right), \quad \text { s.t } 2 x_{1}+x_{2} \leq 3, \quad 0 \leq x_{i} \leq 1, \quad i=1,2 \text {. }
$$

The maximum point of $P$ is $\left(x^{0}\right)^{T}=\left(x_{1}^{0}, x_{2}^{0}\right)=(1,1)$ which is degenerate; there are two optimal bases corresponding to $x^{0}$ that imply, in the standard form,

$$
x_{B_{1}}^{T}=\left(x_{1}, x_{2}, y_{2}\right) \quad \text { and } \quad x_{B_{2}}^{T}=\left(x_{1}, x_{2}, y_{3}\right) .
$$

The corresponding optimal dual solutions are:

$$
c_{B_{1}}^{T} B_{1}^{-1}=(1,0,1) \quad \text { and } \quad c_{B_{2}}^{T} B_{2}^{-1}=(3,1,0),
$$

respectively. Now, let us consider the perturbed problem $P(\varepsilon)$ obtained by posing

$$
\left(\varepsilon_{1}, \varepsilon_{2}, \varepsilon_{3}\right)=\left(\frac{1+(-1)^{n}}{n}, 0, \frac{1-(-1)^{n}}{n}\right), \quad n \in \mathbb{N} \backslash\{0\} .
$$

Obviously, $\lim _{\varepsilon \downarrow 0}\left(\varepsilon_{1}, \varepsilon_{2}, \varepsilon_{3}\right)=(0,0,0)$. If $n$ is even, then the maximum point of $P(\varepsilon)$ in standard form is $\left(x_{1}=1-\frac{2}{n}, x_{2}=1, y_{1}=0, y_{2}=0\right.$, $\left.y_{3}=\frac{4}{n}\right)$ and the dual optimal solution is $\lambda=(3,1,0)$; if $n$ is odd, the maximum point of $P(\varepsilon)$ is $\left(x_{1}=1, x_{2}=1-\frac{2}{n}, y_{1}=0, y_{2}=\frac{2}{n}, y_{3}=0\right)$ and the dual optimal solution is $\lambda=(1,0,1)$. Hence, when $n \rightarrow+\infty$, the limit of the dual solution does not exist. 
Let $E \subseteq \mathbb{R}_{+}^{m}$ be the set where the perturbation parameter $\varepsilon$ runs. The following result holds.

Proposirion 3.2: Every accumulation point of the set $\Lambda:=\{\lambda(\varepsilon): \varepsilon \in E\}$ is a vertex of $E$

Proof: Let $\tilde{\lambda}$ be an accumulation point of $\Lambda$; hence there exists a sequence $\left\{\lambda\left(\varepsilon_{n}\right)\right\}_{n=1}^{+\infty}$ converging to $\tilde{\lambda}$. Therefore, for every $n \in \mathbb{N} \backslash\{0\}$ there exists a problem $P\left(\varepsilon_{n}\right)$ such that $\lambda\left(\varepsilon_{n}\right)$ is an optimal solution of $P^{*}\left(\varepsilon_{n}\right)$ or equivalently there exists a base $B_{n}$ such that $\lambda^{T}\left(\varepsilon_{n}\right)=c_{B_{n}}^{T} B_{n}^{-1}$. Let us consider a subsequence of $\left\{\lambda\left(\varepsilon_{n}\right)\right\}_{n=1}^{+\infty}$, call it $\left\{\lambda\left(\varepsilon_{n_{k}}\right)\right\}_{k=1}^{+\infty}$, such that to every index $k$ corresponds the same base $B$; hence we have that:

$$
\tilde{\lambda}^{T}=\lim _{k \rightarrow+\infty} \lambda^{T}\left(\varepsilon_{n_{k}}\right)=\lim _{k \rightarrow+\infty} c_{B_{n_{k}}}^{T} B_{n_{k}}^{-1}=c_{B}^{T} B^{-1},
$$

i.e., $\tilde{\lambda}$ is a vertex of $F$.

\section{AN INDUSTRIAL PROBLEM}

Let us recall the industrial problem that can be classically represented by (1.1). If an industry produces $n$ goods by employing $m$ resources in the transformation process, then the following definitions are given:

$x^{T}=\left(x_{1}, \ldots, x_{n}\right)$ is the vector of outputs; i.e., $x_{j}$ is the unknown quantity of $j$-th good to be produced, $j=1, \ldots, n$;

$c^{T}=\left(c_{1}, \ldots, c_{n}\right)$ is the vector of profits; i.e., $c_{j}$ represents the profit resulting from the production and the selling of one unit of the $j$-th good, $j=1, \ldots, n$;

$b^{T}=\left(b_{1}, \ldots, b_{m}\right)$ is the vector of input quantities; i.e., $b_{i}$ is the quantity available of the $i$-th resource, $i=1, \ldots, m$;

$A=\left(a_{i j}\right), i=1, \ldots, m ; j=1, \ldots, n$ is the technological matrix; i.e., $a_{i j}$ is the consumption of the $i$-th resource necessary to produce one unit of the $j$-th good.

If we assume that the industry is a linear system, then it is evident that the problem of maximum profit has the formulation (1.1).

If we suppose that the industry may sell the resources directly instead of transforming them, then an alternative problem naturally arises. This is represented by the dual (1.2), where $\lambda^{T}=\left(\lambda_{1}, \ldots, \lambda_{m}\right)$ is the vector of prices of the $m$ resources, and the $j$-th constraint means that the direct selling of all the resources, which are employed to produce one unit of the $j$-th good, 
gives an economic result not worse than that implied by the production of one unit of the $j$-th good. It is interesting to consider those vectors $\lambda$ which minimize the total income deriving from the direct selling of the resources: they are the shadow prices. The comparison between the optimal solution $\lambda^{0}$ of (1.2) and the real prices of the resources, call them $p=\left(p_{1}, \ldots, p_{m}\right)$, leads us to important information on how the resources are exploited in the industry. Such a comparison is determined by setting up the ratios $\frac{\lambda_{i}}{p_{i}}$, $i=1, \ldots, m$ where the critical value is of course 1 .

The remark made in [3] points out a shortcoming of the classic model: if $x^{0}$, maximum point of (1.1), strictly verifies one of the constraints on the consumption of the resources, for example the $r$-th, then $\lambda_{r}^{0}$ is zero. Since $\lambda_{r}^{0}$ is interpreted as the value of one unit of the $r$-th resource, when exploited in the production process, we should conclude that the $r$-th resource, even if used in a positive quantity, has zero value. In other words, in the classic model the value of a resource not completely expended in the optimal production strategy $x^{0}$ is set to zero, while in the real situation the positive use of a resource implies a non-zero value of it.

The proposal of Dantzig and Jackson for overcoming this inadequacy is based on a perturbation method. Example 3.1 shows the possibility that the perturbation method does not work; in fact, even if all the variable elements appearing in the primal problem converge, it may happen that the corresponding dual solutions don't converge; there are only accumulation points. Since an optimal solution of the dual problem is assumed to be a shadow prices vector, the non-existence of the limit implies the impossibility of a correct evaluation of such prices. If we consider the accumulation points of set $\Lambda$ defined in proposition 3.2, we obtain some elements (the vertices) of the face $F$ of the feasible region of the dual problem; therefore the perturbation method does not improve the evaluation already obtained.

The remark of Dantzig and Jackson about the inadequacy of the classic model is interesting and appropriate, but the proposed rectification is not; in fact it does not improve the direct evaluation of the dual solutions. In [3] the inadequacy of the classic model and the subjective resolution of such inadequacy are merged, while the correct way of dealing with the problem is to keep the two aspects apart.

\section{A MECHANiCAL PROBLEM}

The linear model and the Duality Theory can be applied in many fields. In this section we discuss their use in the analysis of a classic and elementary 
mechanical problem. It is important to observe that for such a problem the analysis is suggested by the mathematical formulation of the dual problem; nevertheless, in this case too the dual variables turn out to give important information.

We consider an arbitrary elastic static system $S$ supported on edges (for example a string, a rod or an elastic beam) and choose on it $n$ points, $P_{1}, \ldots, P_{n}$. At every of these points we apply a force; hence we have $n$ forces $F_{1}, \ldots, F_{n}$, where, for every $j=1, \ldots, n, F_{j}$ is expressed as the product between the intensity of the force, supposed equal to 1 , and a multiplicative coefficient $x_{j}$. Moreover, we consider $m$ other points $Q_{1}, \ldots, Q_{m}$ on $S$ (not necessarily coincident with the previous $n$ points), and we are interested in observing the displacements (sags) involved in the $m$ points $Q_{1}, \ldots, Q_{m}$ by the forces $F_{1}, \ldots, F_{n}$ applied at $P_{1}, \ldots, P_{n}$.

Hence we denote by:

$A=\left(a_{i j}\right), i=1, \ldots, m ; j=1, \ldots, n$ the matrix of coefficients of influence; i.e., $a_{i j}$ is the displacement of $Q_{i}$ under the action of a unit force applied at $P_{j}$;

$b^{T}=\left(b_{1}, \ldots, b_{m}\right)$ the vector of the maximum allowed displacements in $Q_{1}, \ldots, Q_{m}$.

We assume that the forces and the displacements are parallel to one and the same direction; moreover, we assume that $S$ is a linear system:

1) under the combined action of two systems of forces the corresponding displacements are added together;

2) when the magnitude of all the forces are multiplied by one and the same real number, then all the displacements are multiplied by the same number.

Under these assumptions, the problem of maximum charge supportable by system $S$ has the following formulation:

$$
\left\{\begin{array}{l}
\max \left(x_{1}+\ldots+x_{n}\right) \\
a_{i 1} x_{1}+\ldots+a_{i n} x_{n} \leq b_{i}, \quad i=1, \ldots, m \\
x_{j} \geq 0, \quad j=1, \ldots, n
\end{array}\right.
$$

i.e., problem (1.1) with $c_{j}=1, j=1, \ldots, n$.

Consider a hypothetical system of forces, alternative to the given one, applied at the points where the displacements are estimated, i.e., $Q_{1}, \ldots, Q_{m}$ instead of $P_{1}, \ldots, P_{n}$; for every $i=1, \ldots, m$, define the variable $\lambda_{i}$ as the intensity of a (hypothetically existing) force per unit displacement. In 
other words, $\lambda_{1}, \ldots, \lambda_{m}$ are hypothetical forces, applied simultaneously at $Q_{1}, \ldots, Q_{m}$, respectively; each of them involves an unit displacement at every of these points.

A comparison between the set of actual forces and the hypothetical one turns out to be interesting. Such a comparison will be made at every point $P_{j}, j=1, \ldots, n$, and under the same displacement: for every $i=1, \ldots, m$, $\lambda_{i} a_{i j}$ is the hypothetical charge applied directly at $Q_{i}$; hence, for every $j=1, \ldots, n, \sum_{i=1}^{m} \lambda_{i} a_{i j}$ represents the sum of hypothetical charges at $Q_{1}, \ldots, Q_{m}$ to be compared with the given unit charge applied at $P_{j}$. The hypothetical system of forces would be not worse than the given one iff the above hypothetical charge was greater than or equal to the unit charge considered in the assigned system:

$$
\lambda_{1} a_{1 j}+\lambda_{2} a_{2 j}+\ldots+\lambda_{m} a_{m j} \geq 1, \quad j=1, \ldots, n
$$

i.e., every solution $\lambda^{T}=\left(\lambda_{1}, \ldots, \lambda_{m}\right)$ of (5.2) is - from the point of view of the charge supported by the system - not worse than the given set of forces.

Obviously, among all these solutions, it is interesting to know a critical one, i.e. a solution implying the minimum value of the total charge which can be achieved in the system alternative to $S$. Since $\lambda_{i}$ is the force per unit displacement and $b_{i}$ the maximum displacement allowed at $Q_{i}, b_{i} \lambda_{i}$ represents the intensity of force (i.e., the charge) hypothetically allowed at $Q_{i}$. Hence, the above total charge is given by $b_{1} \lambda_{1}+\ldots+b_{m} \lambda_{m}$ and the "alternative" problem is

$$
\left\{\begin{array}{l}
\min \left(b_{1} \lambda_{1}+\ldots+b_{m} \lambda_{m}\right) \\
\lambda_{1} a_{1 j}+\ldots+\lambda_{m} a_{m j} \geq 1, \quad j=1, \ldots, n, \\
\lambda_{i} \geq 0, \quad i=1, \ldots, m
\end{array}\right.
$$

which turns out to be the dual of (5.1).

In the industrial problem it is interesting to compare the elements of an optimal dual solution, i.e. the shadow prices, with the real prices. In the mechanical problem an analogous comparison is not possible because of the absence, in the given system, of actual forces associated to the points $Q_{1}, \ldots, Q_{m}$, when they do not coincide with $P_{1}, \ldots, P_{n}$.

On the contrary, following the reasoning of Section 2, there is a perfect analogy with the interpretation of dual variables as velocities. For the sake of simplicity, suppose that the minimum point $\lambda^{0}$ of (5.3) is unique; then the $i$-th component of this dual optimal solution gives the variation, i.e., the 
instantaneous velocity, of the maximum charge with respect to the variation of the allowed displacement in $Q_{i}$. This interpretation gives an important information: let us suppose, for example, that we want to augment the total charge and that $\lambda_{r}^{0}>\lambda_{i}^{0}, i \neq r ; i, r=1, \ldots, m$; the dual alternative model indicates that it is beter to increase the intensity of force in $Q_{r}$ rather than in $Q_{i}$, because $Q_{r}$ is a point with greater velocity. Obviously, if $n=m$ and the points $P_{1}, \ldots, P_{n}$ coincide respectively with $Q_{1}, \ldots, Q_{m}$, the above information concerns with the given real system and not the hypothetical one.

Finally, if the maximum point $x^{0}$ of (5.1) strictly verifies the $r$-th constraint, then $\lambda_{r}^{0}=0$; that is, $\lambda_{r}^{0}$ is null if at point $Q_{r}$ the maximum allowed displacement is not attained by the optimal solution. If we consider $\lambda_{r}^{0}$ as the velocity of variation, then this latter is the correct interpretation; if we define $\lambda_{r}^{0}$ as the variation of maximum charge with respect to the alteration of $b_{r}$, then this variation is not necessarily zero when $a_{r 1} x_{1}^{0}+\ldots+a_{r n} x_{n}^{0}<b_{r}$. The shortage of the model, like in the industrial case, consists in considering valid the passage from the mathematical interpretation $\left(\lambda_{i}^{0}, i=1, \ldots, m\right.$ are derivatives, i.e. velocities) to the industrial or mechanical one (shadow prices or changes of maximum charge) not only when $\lambda_{r}^{0}>0$, but also when $\lambda_{r}^{0}=0$. Hence the same considerations about the method for overcoming this lack in the industrial case apply to the mechanical one.

\section{REFERENCES}

1. F. Arcangeli, Some remarks on dual variables and shadow prices in linear programming, Proceeding of the Working Day on "Mathematical Optimization: Theory, Methods and Applications", Verona, December 1992, K.-H. Elster and C. Sutti (eds.), 1993, pp. 23-27.

2. G. B. Dantzig, Linear programming and extensions, Princeton University Press, Princeton N. Y., 1963.

3. G. B. DANTZIG and P. L. JACKSON, Pricing underemployed capacity in a linear economic model, in "Variational inequalities and complementarity problems", R. W. Cottle, F. Giannessi and J.-L. Lions (eds.), J. Wiley, 1980, pp. 127-134.

4. M. Volpato, I prezzi ombra come fattori di decisione, in Studi e Modelli di Ricerca Operativa, M. Volpato (ed.), UTET, Torino, 1971, pp. 122-138. 